Henry Antonio

Mendoza Tolosa*

Carlos Andrés Yanes Guerra**:

Universidad Católica de Colombia, Bogotá, Colombia.

Recibido: 12 de junio de 2013 Concepto de evaluación: 17 de febrero de 2014 Aprobado: 17 de marzo de 2014

Artículo de investigación DOI: $h$ ttp://dx.doi.org/10.14718/ revfinanzpolitecon.2014.6.1.2

(C) 2014 Universidad Católica de Colombia. Facultad de Ciencias Económicas y Administrativas. Todos los derechos reservados.

*Magister en ciencias económicas con énfasis en economía y gestión pública. Docente e investigador de la Facultad de Ciencias Económicas y Administrativas, Universidad Católica de Colombia.Dirección de correspondencia: Facultad de Ciencias Económicas y Administrativas, Universidad

Católica de Colombia, cr. 13 \# 47-49, Bogotá, Colombia. Correo electrónico: hamendoza@ucatolica.edu.co

**Magister en economía. Docente e investigador de la Facultad de Ciencias Económicas y Administrativas, Universidad

Católica de Colombia. Dirección de correspondencia: Facultad de Ciencias Económicas y Administrativas, Universidad Católica de Colombia,

cr. 13 \# 47-49, Bogotá,

Colombia. Correo electrónico: cayanes@ucatolica.edu.co
Finanz. polit. econ., ISSN: 2248-6046, Vol. 6, No. 1, enero-junio, 2014, pp. 23-41

Edición Especial - Economía Regional

\section{Impacto del gasto público en la dinámica económica regional ${ }^{1}$}

\section{RESUMEN}

Se estudia el impacto que tienen el gasto y la inversión pública en el crecimiento económico de los departamentos en Colombia, utilizando los resultados de las cuentas nacionales para el periodo 2000-2011; se estima la cuenta de producción departamental por rama de actividad con el fin de identificar la estructura productiva de dichas regiones, así como sus cambios a lo largo del tiempo, y se consolida información de la formación bruta de capital fijo público, datos con los cuales se especifica un modelo estadístico que estime dichos efectos. Entre las diferentes alternativas instrumentales, se diseña un modelo de panel de datos de efectos fijos que refleja de manera adecuada las diferencias existentes entre los departamentos y captura mejor el impacto del gasto y la inversión con la temporalidad e información disponible. Los resultados indican que el gasto y la inversión públicos inciden directamente en la dinámica económica departamental y se evidencia que su efecto tiende a ser mejor aprovechado por las economías grandes más ricas en mayor medida que por las economías departamentales pequeñas y pobres.

Palabras clave: dinámica económica regional, gasto público, PIB departamental.

JEL: H72, R10, R50

\section{The Impact of Public Spending on Regional Economic Dynamics}

\section{ABSTRACT}

The impact that public spending and investment have upon economic growth in the departments of Colombia is examined using the results of national accounts for the years 2000-2011. Figures for departmental production by activity, along with change over the period and information for the gross public capital are brought together to create a statistical model to assess effects. A data panel model is chosen to relate the existing differences between departments and compare the impact of spending and investment between departments using the available information. Results indicate that public spending and investment play an important role in departmental economic dynamic and that its effect is greater in larger and wealthier departments. GDP.

Keywords: Regional economic dynamic, public spending, departmental

$\overline{1}$ El presente trabajo hace parte de las actividades de investigación desarrollada por los autores en la Facultad de Ciencias Económicas y Administrativas. 


\section{Impacto do gasto público na dinâmica econômica regional}

\section{RESUMO}

Neste artigo, estuda-se o impacto que o gasto e o investimento público têm no crescimento econômico dos estados na Colômbia, utilizando os resultados das contas nacionais para o período 2000-2011; estima-se a conta de produção estadual por ramo de atividade com o objetivo de identificar a estrutura produtiva dessas regiões, bem como suas mudanças ao longo do tempo e consolida-se informação da formação bruta de capital fixo público, dados com os quais se especifica um modelo estatístico que estime esses efeitos. Entre as diferentes alternativas instrumentais, desenha-se um modelo de painel de dados de efeitos fixos que reflita de maneira adequada as diferenças existentes entre os estados e captura melhor o impacto do gasto e do investimento com a temporalidade e informação disponível. Os resultados indicam que o gasto e o investimento públicos incidem diretamente na dinâmica econômica estadual e evidencia-se que seu efeito tende a ser melhor aproveitado pelas economias grandes mais ricas em maior medida que pelas economias estaduais pequenas e pobres.

Palavras-chave: dinâmica econômica regional, gasto público, PIB estadual. 


\section{INTRODUCCIÓN}

El presente trabajo tiene por objeto identificar la influencia del gasto y la inversión pública en la dinámica económica de los departamentos en Colombia, durante el periodo 2000-2011. A efectos de este estudio, el gasto público representa las erogaciones efectuadas por los gobiernos departamentales en bienes y servicios asignados a la comunidad, incluidos los gastos de funcionamiento que se requieren para garantizar su normal funcionamiento. Por su parte, la inversión pública comprende los pagos hechos por estos territorios ${ }^{2}$, orientados a incrementar la formación bruta de capital fijo de los departamentos, a fin de fortalecer su capacidad productiva y competitiva. Estas variables se explican con mayor detalle en el diseño metodológico.

Para tal efecto, el análisis económico y estadístico se adelantará a partir de las cuentas nacionales por departamentos publicadas por el Departamento Administrativo Nacional de Estadística (DANE), las series de gasto público y las de formación bruta de capital fijo de esas regiones. El Producto Interno Bruto (PIB) departamental constituye uno de los referentes principales para conocer la dinámica económica de las regiones, resultado de la distribución del PIB nacional por actividades económicas con base en una metodología centralizada que emplea los indicadores líderes disponibles de los diferentes sectores productivos de cada región. Sin embargo, este sistema de información no está completamente desarrollado en el país, en buena parte como resultado de la carencia que tiene nuestro sistema estadístico de información, registros administrativos e indicadores de comportamiento económico sectorial y productivo. Lo anterior se convierte en una limitante al momento de profundizar en los determinantes de la dinámica económica regional.

De forma alternativa, progresivamente ha aumentado la demanda de estudios regionales, así como de información estadística y de análisis

2 En este documento se hará referencia indistintamente a las regiones, territorios, entes territoriales, etc., haciendo alusión a los departamentos como las unidades estadísticas objeto de estudio. de tipo territorial oficial estandarizada que expresa los requerimientos de factores y productos en cada región, identifica fuentes y usos de la inversión en el sector real, la competitividad y desarrollo de los entes territoriales, cuyas fortalezas competitivas estructurales no son evidentes en el sistema de información disponible actualmente. Por lo tanto, responder a la pregunta de si el gasto y la inversión públicos de los gobiernos territoriales han contribuido a fortalecer la dinámica económica de los departamentos se convierte en la cuestión por resolver.

En respuesta al problema planteado de conformidad con los estudios que sobre este tema aborda la literatura, la disponibilidad temporal de series asociadas y la cobertura de los datos de estudio, este artículo se consolidó mediante un ejercicio de panel longitudinal de efectos fijos para el periodo 2000-2011, combinando la información estadística departamental disponible y agrupando las regiones de Colombia en tres grupos focales de estudio, según el tamaño de su PIB: economías grandes, medianas y pequeñas.

El presente documento se divide en cuatro secciones. En la primera parte, se presentan una breve descripción del estado del arte en torno a la evidencia que la literatura ofrece en cuanto a los determinantes de la dinámica regional. En la segunda sección se explica la metodología abordada y el camino recorrido en este proceso. Posteriormente, se exponen y explican los resultados obtenidos de acuerdo con el instrumental utilizado y finalmente se discuten en contexto las implicaciones derivadas de los hallazgos asociados al impacto del gasto y la inversión pública en el comportamiento económico de las regiones.

\section{METODOLOGÍA}

El diseño metodológico para identificar el impacto del gasto y la inversión pública en la dinámica económica de las regiones se enfocó en diseñar un modelo que determine el tipo de relación existente a partir de la información disponible de las cuentas nacionales departamentales 2000-2011, 
el cual se reforzó con estimaciones cuantitativas de agregados de la cuenta de producción y generación del ingreso regional, así como la compilación de datos asociados a los gastos de los departamentos en formación bruta de capital fijo, los cuales se constituyeron en los insumos del diseño del instrumento de cálculo.

Para tal efecto, la primera parte de esta sección presenta los referentes teóricos contemplados en este documento, de conformidad con el estado del arte de este tema, y en la segunda parte se describe el proceso metodológico adelantado.

\section{Referentes conceptuales}

En Colombia, los estudios sobre dinámica de las economías regionales se han orientado principalmente a analizar el impacto de la distribución del ingreso y las asimetrías regionales con base en la comparación de datos y el contraste de diversas fuentes de información (Ardila, 2004). Investigaciones alternativas han analizado la convergencia económica del ingreso regional hacia un referente empírico nacional (Moncayo, 2007); otros estudios han buscado identificar si hay uniformidad en el crecimiento de las regiones o si existen desbalances en su comportamiento, considerando variables exógenas como la migración interna para explicar dicho comportamiento (Sánchez, 2006), en tanto que en el sentido del análisis de convergencia del crecimiento regional se orientan trabajos como el de Pérez (1999) en Chile.

En la literatura para Colombia son escasas las investigaciones que han abordado el impacto que genera el gasto público en el crecimiento económico departamental. En algunos de ellos, (Rocha y Vivas, 1998) concluyeron que la política fiscal tiene un efecto negativo en el crecimiento económico. Así mismo, Rocha y Martínez (2003) evidenciaron que a pesar de la creciente ola de criminalidad y narcotráfico existente por entonces en buena parte de los departamentos, las políticas públicas con impacto social tuvieron un efecto positivo en la actividad económica de las regiones.
Por su parte, Fuentes (2003), mediante un estudio econométrico, describió las implicaciones del gasto público en infraestructura en el crecimiento económico regional de México. Agúndez y Pedraja (1999) analizaron la incidencia del gasto público en el crecimiento regional mediante el estudio de la contribución potencial de la descentralización fiscal, vía ingresos públicos, al desarrollo y crecimiento económico regional. Esta investigación, originada en España, basó su centro de atención en el desarrollo de un modelo que relaciona la distribución de ingresos públicos en una región entre distintos niveles de gobierno con su propio crecimiento económico.

Gamboa y Messmacher (2002) determinaron el impacto del gasto público sobre el crecimiento económico en un ámbito de apertura económica. En su artículo los autores determinan, entre otros aspectos, la incidencia del gasto público en la desigualdad de prestación de servicios estatales en México, de lo cual se concluye que en un ámbito de apertura comercial hay estados que se encuentran en desventaja respecto a otros y por ello el sistema fiscal federal los compensa. Los autores no encontraron evidencia respecto a que un mayor gasto público redunde en un crecimiento económico regional más vigoroso. A propósito del crecimiento regional y el impacto que sobre este se genere, como resultados de descensos importantes en la formación bruta de capital público, en el marco de la Conferencia Internacional de Ciencia Regional (España) se estableció que las caídas sistemáticas de la inversión pública local tienen efectos negativos en el producto de todas las regiones (Márquez y Ramajo, 2012).

En América Latina, estudios como el de Baquero, Rocha y Ortega (2006) aportan nuevos elementos de discusión respecto a la relación entre la política de gasto rural y el desarrollo económico de los países. Estos autores llegaron a dos principales conclusiones: la primera, que el nivel de gasto importa, pero no tanto como su composición; la segunda, que en América Latina y el Caribe el gasto público ha mostrado un sesgo favorable a las élites económicas. 
En general, se aprecia que en los citados estudios es común el uso limitado de las cuentas nacionales departamentales, en particular por el escaso alcance de sus resultados. Colombia no es la excepción y esto obedece en gran medida a las restricciones en la oferta de información del sistema estadístico territorial, así como de indicadores de coyuntura que evidencian la dinámica productiva y la asignación de recursos a las entidades territoriales. Otra limitante que muestra la literatura revisada en la construcción de este tipo de estudios es la disponibilidad de series de tiempo de las cuentas departamentales suficientemente extensas que permitan hacer análisis de largo plazo sobre el comportamiento de sus economías, aspecto que conduce a afirmar que en la actualidad son pocos los estudios que permiten establecer los efectos del gasto público en el crecimiento económico de las regiones (Vallecilla, 2012).

Partiendo de esta reseña relacionada con los trabajos que se han hecho recientemente y que son punto de partida del problema en comento, se da paso a la descripción del proceso metodológico adelantado en torno a los temas que atañen al presente estudio.

\section{Diseño metodológico}

Las cuentas departamentales elaboradas por el DANE se obtienen a partir del método centralizado o descendente. Este método consiste en distribuir entre todos los departamentos los agregados nacionales relacionados con la producción, por medio del uso de indicadores estructurales para cada actividad económica. Con esto se busca la coherencia de niveles, evoluciones y resultados de las cuentas nacionales anuales, que son su referente (DANE, 2010). No obstante, para atender el objeto de investigación este sistema contable no cubre el conjunto de cuentas que permitan identificar los distintos determinantes del crecimiento, la generación de ingreso y la utilización del mismo.

En tal sentido, el proceso metodológico se cubrió en dos etapas combinando alternativamente métodos cuantitativos de medición basada en esta- dística observada con técnicas de inferencia estadística de frontera. En efecto, en la primera etapa se adelantó un ejercicio de cuantificación de los agregados de la cuenta de producción, a fin de obtener series de producción y consumo intermedio por departamento. Mediante dichos agregados se identificaron los requerimientos técnicos de la función de producción de cada entidad territorial que complementarán el modelo cuantitativo formulado. En la segunda etapa se estimó un modelo tipo panel, cuyas especificaciones se orientaron a estimar el impacto del gasto y la inversión pública en el producto regional. En su construcción se emplearon los resultados de las series de las cuentas departamentales, la cuenta de producción por departamento y la serie compilada de gasto público en formación bruta de capital fijo.

Para calcular la cuenta de producción por departamentos se utilizaron los resultados de los coeficientes técnicos por rama de actividad derivados de la matriz oferta-utilización de las cuentas nacionales, año por año para la serie 2000-2011 y las estructuras de los establecimientos obtenidas de las cuentas departamentales. En tal sentido, la producción departamental se infiere a partir de la relación:

$P i t=P t * \frac{V A B i t}{V A B t}$

Donde:

Pit $=$ producción del departamento $i$ en el periodo $t$

$\mathrm{P} t=$ producción total de la economía en el periodo $t$

VABit $=$ valor agregado bruto del departamento $i$ en el periodo $t$

$\mathrm{VAB} t=$ valor agregado bruto total de la economía en el periodo $t$

$i=1,2, \ldots 33$ (Bogotá tiene tratamiento como departamento)

$t=\quad 2000,2001, \ldots 2011$ 
De conformidad con [1], se deduce que el consumo intermedio departamental corresponde a:

$$
\text { CIit }=\text { Pit }- \text { VABit }
$$

Donde:

Pit $=$ producción del departamento $i$ en el periodo $t$

VABit $=$ valor agregado bruto del departamento $i$ en el periodo $t$

Clit $=$ consumo intermedio del departamento $i$ en el periodo $t$

Teniendo en cuenta [1] y [2], a nivel de rama de actividad por departamento se tiene que:

$\mathrm{P} k i t=P i t * \frac{V A B k i t}{V A B i t}$
Donde:

Pkit $=$ producción de la rama de actividad $k$ en el departamento $i$ durante el periodo $t$

Pit $=$ producción del departamento $i$ durante el periodo $t$

VABkit $=$ valor agregado bruto de la rama de actividad $k$ en el departamento $i$ durante el periodo $t$

VABit $=$ valor agregado bruto del departamento $i$ durante el periodo $t$

$k=1,2, \ldots .9$

Finalmente, de [3] se deduce que:

CIkit $=$ Pkit - VABkit

[4]

Tabla 1.

Cuenta de producción departamental por rama de actividad económica, 2009 (ejemplo de caso).

Cifras en miles de millones de pesos

\begin{tabular}{|c|c|c|c|c|c|c|c|c|c|c|}
\hline 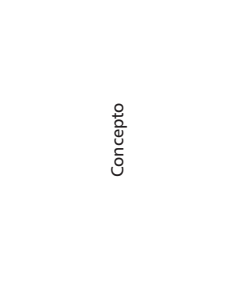 & 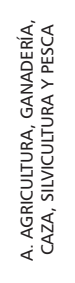 & 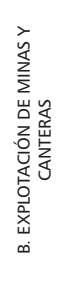 & 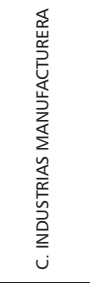 & 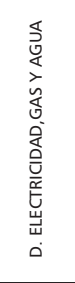 & 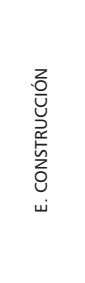 & 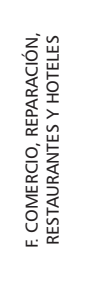 & 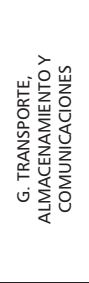 & 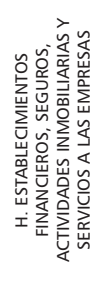 & 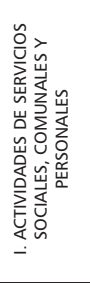 & 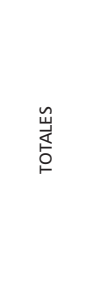 \\
\hline \multicolumn{11}{|c|}{ Bogotá D. C. } \\
\hline $\begin{array}{l}\text { Valor agregado por } \\
\text { rama de actividad }\end{array}$ & - & 244 & 14.683 & 3.805 & 9.178 & 17.469 & 9.064 & 42.576 & 22.924 & 119.943 \\
\hline $\begin{array}{l}\text { Participación valor } \\
\text { agregado por rama de } \\
\text { actividad }\end{array}$ & 0,0 & 0,0 & 0,1 & 0,0 & 0,1 & 0,1 & 0,1 & 0,4 & 0,2 & 1,0 \\
\hline $\begin{array}{l}\text { Producción estimada } \\
\text { por rama de actividad }\end{array}$ & - & 453 & 27.247 & 7.061 & 17.031 & 32.417 & 16.820 & 79.007 & 42.539 & 222.574 \\
\hline $\begin{array}{l}\text { Consumo intermedio } \\
\text { por rama de actividad }\end{array}$ & - & 209 & 12.564 & 3.256 & 7.853 & 14.948 & 7.756 & 36.431 & 19.615 & 102.631 \\
\hline \multicolumn{11}{|c|}{ Antioquia } \\
\hline $\begin{array}{l}\text { Valor agregado por } \\
\text { rama de actividad }\end{array}$ & 4.376 & 1.919 & 9.351 & 3.675 & 6.651 & 8.869 & 4.141 & 14.322 & 9.264 & 62.568 \\
\hline $\begin{array}{l}\text { Participación valor } \\
\text { agregado por rama de } \\
\text { actividad }\end{array}$ & 0,1 & 0,0 & 0,1 & 0,1 & 0,1 & 0,1 & 0,1 & 0,2 & 0,1 & 1,0 \\
\hline $\begin{array}{l}\text { Producción estimada } \\
\text { por rama de actividad }\end{array}$ & 8.036 & 3.524 & 17.172 & 6.749 & 12.214 & 16.287 & 7.604 & 26.301 & 17.012 & 114.899 \\
\hline $\begin{array}{l}\text { Consumo intermedio } \\
\text { por rama de actividad }\end{array}$ & 3.660 & 1.605 & 7.821 & 3.074 & 5.563 & 7.418 & 3.463 & 11.979 & 7.748 & 52.331 \\
\hline \multicolumn{11}{|c|}{ Boyacá } \\
\hline $\begin{array}{l}\text { Valor agregado por } \\
\text { rama de actividad }\end{array}$ & 2.313 & 1.382 & 1.924 & 699 & 971 & 1.614 & 944 & 1.208 & 2.146 & 13.201 \\
\hline $\begin{array}{l}\text { Participación valor } \\
\text { agregado por rama de } \\
\text { actividad }\end{array}$ & 0,2 & 0,1 & 0,1 & 0,1 & 0,1 & 0,1 & 0,1 & 0,1 & 0,2 & 1,0 \\
\hline $\begin{array}{l}\text { Producción estimada } \\
\text { por rama de actividad }\end{array}$ & 4.185 & 2.501 & 3.481 & 1.265 & 1.757 & 2.920 & 1.708 & 2.186 & 3.883 & 23.885 \\
\hline $\begin{array}{l}\text { Consumo intermedio } \\
\text { por rama de actividad }\end{array}$ & 1.872 & 1.119 & 1.557 & 566 & 786 & 1.306 & 764 & 978 & 1.737 & 10.684 \\
\hline
\end{tabular}

Fuente: elaboración de los autores, con base en la cuentas nacionales del DANE. 
Donde:
Clkit $=$ consumo intermedio de la rama de actividad $k$ en el departamento $i$ durante el periodo $t$

Un resultado ilustrativo del procedimiento adoptado se aprecia en la tabla 1.

Por otra parte, el vector de gasto público para el periodo de estudio fue conformado con el valor de la provisión gubernamental de bienes y servicios de carácter colectivo e individualizable identificados de las cuentas nacionales departamentales por:

$G i t=A P i t+E i t+E S i t$

Donde:

$$
\begin{array}{ll}
\text { Git }=\quad \begin{array}{l}
\text { gasto público del departamento } i \\
\text { durante el periodo } t
\end{array} \\
\text { APit }=\quad \begin{array}{l}
\text { gastos de administración pública y } \\
\text { defensa; seguridad social de afilia- } \\
\text { ción obligatoria del departamento } \\
i \text { durante el periodo } t
\end{array} \\
\text { Eit }=\quad \begin{array}{l}
\text { gastos en educación de no mercado del } \\
\text { departamento } i \text { durante el periodo } t
\end{array} \\
\begin{array}{l}
\text { gastos de actividades de asocia- } \\
\text { ciones n.c.p.; actividades de espar- } \\
\text { cimiento, culturales, deportivas y } \\
\text { otras de servicios de no mercado del } \\
\text { departamento } i \text { durante el periodo } t
\end{array}
\end{array}
$$

La estimación de Git responde entonces a la producción de gobierno desde la óptica del Sistema de Cuentas Nacionales y por tanto no considera los subsidios y/o subvenciones específicas focalizadas, por cuanto lo que se busca es identificar el impacto del gasto público de manera independiente a las erogaciones directas hechas por el Estado a diferentes sectores productivos con fines de política económica. Los datos de formación bruta de capital fijo e inversión social en transferencias ejecutados por los gobiernos locales se consolidaron de la información que sobre este concepto dispone el Departamento Nacional de Planeación en su página de Internet.
Con el propósito de facilitar el procesamiento y análisis de los datos departamentales (incluida Bogotá), la información obtenida se organizó en tres grupos iguales de integrantes, de conformidad con la participación media de sus valores agregados durante el periodo de estudio, lo cual determinó la formación de los siguientes conjuntos de departamentos:

1. Economías grandes: Bogotá, Antioquía, Valle, Santander, Cundinamarca, Atlántico, Bolívar, Meta, Boyacá, Tolima y Casanare.

2. Economías medianas: Córdoba, Huila, Cesar, Norte de Santander, Caldas, Nariño, Risaralda, Cauca, Magdalena, La Guajira y Arauca.

3. Economías pequeñas: Quindío, Sucre, Caquetá, Chocó, Putumayo, San Andrés y Providencia, Guaviare, Amazonas, Vichada, Guainía y Vaupés.

En una segunda etapa, la especificación del modelo óptimo que determina el impacto del gasto público en la dinámica económica de los departamentos se constituyó a partir de las bases de datos conformadas para los departamentos (PIB total, producción, consumo intermedio y valor agregado por rama de actividad económica, gasto público colectivo e individualizable, así como los gastos de formación bruta de capital fijo e inversión social en transferencias del mismo sector asignados a estas entidades territoriales).

Para tal efecto, se seleccionó un modelo del tipo panel de datos que hace referencia a un conjunto de observaciones de las que se dispone información en varios periodos. El uso del panel aplica a cada una de las variables constituidas, dado que estas se pueden diferenciar como individuos $(i)^{3}$ y por temporalidad de acuerdo con las series disponibles que van de 2000 hasta 2011 ( $t$ ).

Para desarrollar el análisis de panel longitudinal de la serie, se adelantó una caracterización por cada una de las variables de interés, en la cual para

3 Cada uno de los departamentos, incluyendo a Bogotá, serán tomados como los agentes dinámicos del modelo; los panel data permiten la bidimensionalidad del estudio que contiene información para cada uno de los entes territoriales para la serie de tiempo disponible. 
el crecimiento económico se definió como variable proxy el logaritmo del PIB departamental anual. Por su parte, el gasto público del Gobierno local se representó mediante su logaritmo y la inversión pública se identificó mediante el logaritmo de la formación bruta de capital fijo4.

Disponer de datos con características longitudinales, en términos de tiempo y observaciones, define una base sobre la que es factible eliminar los efectos cíclicos, siendo útil además para probar teorías de comportamiento con enfoque de largo plazo. Particularmente, este tipo de análisis facilita la solución de problemas de endogeneidad ${ }^{5}$ que suelen resultar de variables omitidas que se presentan con periodicidad constante. Así mismo, como cuando se tienen datos de corte transversal, estos modelos permiten identificar efectos que usualmente no se pueden detectar en ese tipo de muestras y por ende es más precisa la estimación de los parámetros (Batalgi, 2008).

Los modelos tradicionales conocidos en la literatura como de corte transversal (solo varían a través de individuos), así como los de series de tiempo que solo cambian por la temporalidad definida, no permiten capturar conjuntamente los efectos propios de la heterogeneidad de las variables observadas, que en el caso que nos ocupa corresponden a las particularidades de la estructura económica de cada uno de los departamentos analizados, en tanto que la técnica de panel longitudinal sí. En este caso, los impactos individuales departamentales son invariantes en el tiempo y corresponderán a la capacidad empresarial y organizativa, la eficiencia, el acceso a tecnologías y la territorialidad, entre otros. Los efectos temporales recaen por igual en cada una de las observaciones como choques macroeconómicos (inflación, tasas de interés, leyes y demás cambios institucionales, etc.).

$4 \quad$ Este trabajo consideró como referente de estudio el valor de la inversión pública, y lo hizo equivalente al gasto público orientado a crear formación bruta de capital fijo (infraestructura, vivienda, maquinaria, equipo, etc.)

5 La endogeneidad se evidencia en dos casos específicos. El primero, cuando existe una alta correlación entre las variables explicativas del modelo con el término estocástico del error y esta no se detecta. La segunda, cuando hay implícita una doble causalidad o relación entre las variables explicativas y la variable independiente y no es fácil determinar cuál de ellas dentro del orden de la causalidad afecta a la(s) otra(s).
En suma, dicha metodología permite analizar los efectos individuales específicos y los temporales de manera simultánea de acuerdo con el objeto de estudio. En este sentido, el siguiente paso por resolver consiste en elegir entre un panel data de efectos fijos o uno que modele efectos aleatorios.

\section{Modelo de efectos fijos}

Este modelo toma en consideración los aspectos o características de las variables que solo cambian por individuos - en este caso, los departamentos-, teniendo en cuenta cualidades diferentes entre estos. Algunas de esas características están implícitas en el tamaño o en la dinámica de sus economías y particularidades regionales; el supuesto incorporado es que es posible encontrar grandes variaciones entre la economía regional grande, como el Valle, y una economía de la región Caribe, como La Guajira6. El modelo de panel de datos de efectos fijos alternativo definido es:

$$
\begin{aligned}
& \text { Crecimiento }_{i t}=\varphi_{1} \text { Gasto }_{i t}+\varphi_{2} \text { Formación }_{i t} \\
& +\alpha_{i}+\varepsilon_{i t}
\end{aligned}
$$

Donde:

$\mathrm{i}=$ departamentos de estudio

$\mathrm{t}=$ temporalidad que va desde 2000 hasta 2011

$\alpha_{i}=$ parámetro asociado a cada departamento

Crecimiento $_{i t}=$ logaritmo del PIB departamental

Gasto $_{i t}=$ logaritmo del gasto público departamental

$\varphi_{2}$ Formacion $_{i t}=$ logaritmo de la formación bruta de capital fijo

$\varphi_{1}=$ coeficiente para medida de proporción de efecto

$\varepsilon_{i t}=$ Término de error

$6 \quad$ Cabe recordar que en el modelo de efectos fijos, tal efecto es invariante entre individuos o departamentos, como es este caso. Por ejemplo, una de esas características puede ser su extensión geográfica, la calidad de su tierra, la raza predominante, etc. 


\section{Modelo de efectos aleatorios}

Este modelo tiene la misma especificación de un modelo de efectos fijos, pero en este caso se parte del supuesto de homogeneidad en las características de los departamentos; por tanto, aquí no habría diferencia entre cada uno de ellos y por ser un modelo generalizado, en el que el intercepto individual es completamente aleatorio, hace que la varianza sea mínima y los estimadores más eficientes, aunque menos consistentes que en el caso del panel de efectos fijos.

En virtud de la hipótesis de aleatoriedad del intercepto, se considera que en realidad no se tiene certeza del valor que este pueda tener por cada observación y que por tanto su valor giraría en torno a la media global de los departamentos, donde de hecho existe homogeneidad entre todos sus individuos.

El modelo de panel de datos de efectos aleatorios factible por utilizar se define como:

$$
\begin{aligned}
& \text { Crecimiento }_{i t}=\varphi_{1} \text { Gasto }_{i t}+\varphi_{2} \text { Formación }_{i t} \\
& +\alpha_{i}+\varepsilon_{i t}+\mu_{i t}
\end{aligned}
$$

\section{Donde:}

$\alpha_{i}=$ representa el parámetro para cada departamento Crecimiento $_{i t}:=$ logaritmo del PIB departamental Gasto $_{i t}=$ logaritmo del gasto público departamental Formación $_{i t}=$ logaritmo de la formación bruta de capital $\varphi_{1}=$ coeficiente para medida de proporción de efecto $\varepsilon_{i t}=$ término del error por departamento.

$\mu_{i t}=$ error total del modelo

De conformidad con los datos compilados y construidos, así como con la especificación de los modelos alternativos tipo panel data de efectos fijos o aleatorios, a continuación se presentan los resultados descriptivos que se destacan y que están orientados a establecer el efecto del gasto y la inversión pública en el comportamiento de las economías regionales durante el periodo cubierto.

\section{Modelo de efectos fijos, corrección de Arellano-Bond}

Dado que la investigación abarca el uso de panel de datos dinámicos, se asume que por construcción los efectos no observados en estos paneles (errores idiosincráticos) tienen características que no varían en el tiempo (efectos fijos), las cuales identificamos como aquellos aspectos demográficos y geográficos que con regularidad suelen estar correlacionados con las variables del gasto público y la formación bruta de capital. Estos, a su vez, se encuentran correlacionados con los rezagos de la variable dependiente, lo que provee los resultados obtenidos como estimadores inconsistentes.

La idea es corregir la endogeneidad que teóricamente está establecida por la naturaleza de cada una de las variables de nuestro modelo que, si bien se conoce, el crecimiento económico está explicado como se muestra en la ecuación [6]; pero además, este también puede causar y tener relación con la dinámica de la formación bruta de capital y con el mismo gasto público, ya que la causalidad puede ir en ambas direcciones y por tanto nuestros regresores pueden tener relación con el error del modelo.

Luego, si aplicamos rezagos a la variable del crecimiento económico y el uso del método generalizado de momentos, se obtiene:

Crecimiento $_{i t}=\sum_{i=1}^{p} \gamma_{i}$ Crecimiento $_{i, t-j}+\varphi_{1}$ Gasto $_{i t}$

$+\beta_{i t} w_{i t}+\varphi_{2}$ Formación $_{i t}+\alpha_{i}+\varepsilon_{i t}$

Donde:

$\mathrm{i}=$ departamentos de estudio

$\mathrm{t}=$ temporalidad que va desde 2000 hasta 2011

$\alpha_{i}=$ parámetro asociado a cada departamento

$\beta_{\text {it }}=$ vector de parámetros para ser estimado

$\mathrm{w}_{\mathrm{it}}=$ vector de covariables predeterminado

Crecimiento $_{i t}=$ logaritmo del PIB departamental y rezagos

Gasto $_{i t}=$ logaritmo del gasto público departamental

Formacion $_{i t}=$ Logaritmo de la formación bruta de capital fijo

$\varphi_{1}=$ Coeficiente para medida de proporción de efecto

$\varepsilon_{i t}=$ Término de error 
Siguiendo la metodología de Arellano y Bond (1991), el MMG usa los rezagos de las variables para corregir el problema de la endogeneidad, ya que estos toman la propiedad de ser instrumentos válidos y que la varianza del error idiosincrático $\epsilon_{i t}$-que para este caso es conocido como el parámetro - se vuelve constante para cada una de las variables del modelo.
Después de diferenciarlo se tiene lo siguiente:

$$
\begin{aligned}
& \Delta \text { Crecimiento }_{i t}=\Delta \sum_{i=1}^{p} \gamma_{i} \text { Crecimiento }_{i, t-j} \\
& +\Delta \varphi_{1} \text { Gasto }_{i t}+\Delta \beta_{i t} w_{i t}+\Delta \varphi_{2} \text { Formación }_{i t}+\Delta \varepsilon_{i t}
\end{aligned}
$$

La ecuación [8] muestra que mediante la diferenciación de las variables y la aplicación de los rezagos no existe relación entre las variables explicativas y $\epsilon_{i t} \circ \epsilon_{i t-1}$, que finalmente es lo que se requiere para la correcta estimación y cálculo de los efectos del gasto y la formación de capital en el crecimiento económico.

Gráfica 1 .

Dinámica de la variación porcentual del PIB departamental a precios corrientes.

Economías grandes, medianas y pequeñas (2001- 2011)

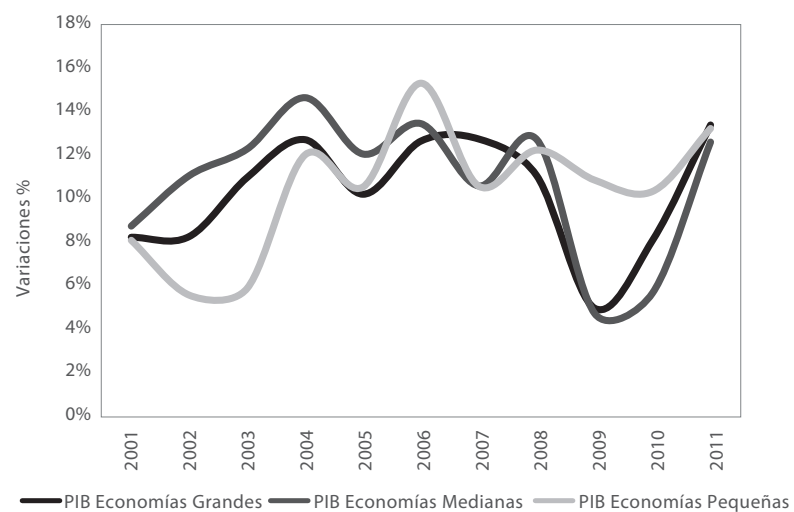

Fuente: elaboración de los autores, con base en las cuentas nacionales del DANE.

Gráfica 2.

Dinámica de la variación porcentual de la producción departamental a precios corrientes.

Economías grandes, medianas y pequeñas (2001- 2011)

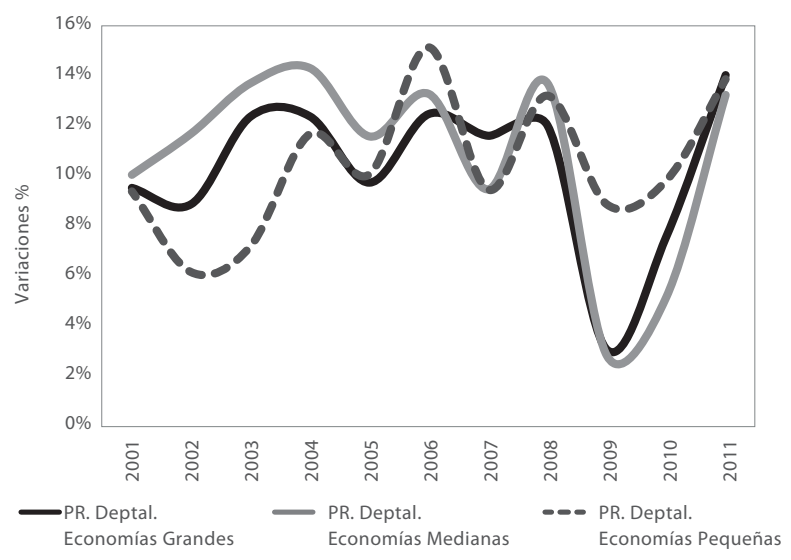

Fuente: elaboración de los autores, con base en las cuentas nacionales del DANE. 


\section{RESULTADOS}

Analizando el PIB en términos absolutos en un contexto descriptivo para los tres grupos focales de departamentos (economías grandes, medianas y pequeñas), se evidencia una relación directa y una tendencia creciente para casi todo el periodo. El ritmo más bajo de crecimiento de la serie se presentó en 2009, consecuente con el efecto generado por la desaceleración del crecimiento económico formado al final de dicha década. En análisis gráfico por variaciones porcentuales muestra esta situación de forma mucho más clara; así mismo, es evidente que las economías pequeñas no se vieron mayormente afectadas por dicha reducción del ritmo (gráficas 1 y 2).

Por otro lado, la dinámica del gasto público fue más volátil en las economías pequeñas, particularmente en 2004 y 2008, años en los que superó el crecimiento de las demás economías siendo su punto más bajo 2002, cuando presentó una leve caída (la única de la serie). Sin embargo, aparentemente este comportamiento no influenció de la misma manera el crecimiento de su producto y PIB, pues en sendos años las economías pequeñas crecieron por debajo de alguno de los otros grupos de estudio. Por su parte,

Gráfica 3.

Gasto público. Economías grandes, medianas y pequeñas a precios corrientes 2001 - 2011 (variaciones porcentuales)

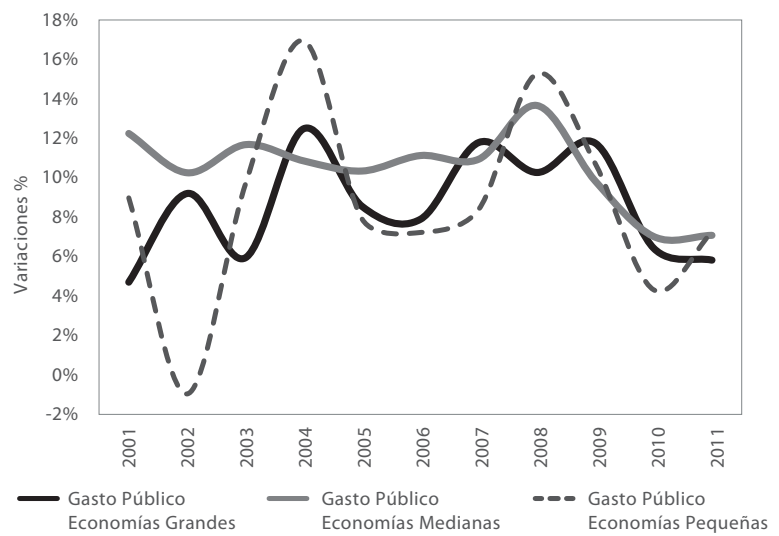

Fuente: elaboración de los autores, con base en las cuentas nacionales del DANE.

Gráfica 4.

Formación bruta de capital fijo público regional a precios corrientes 2000- 2011 (valores absolutos)

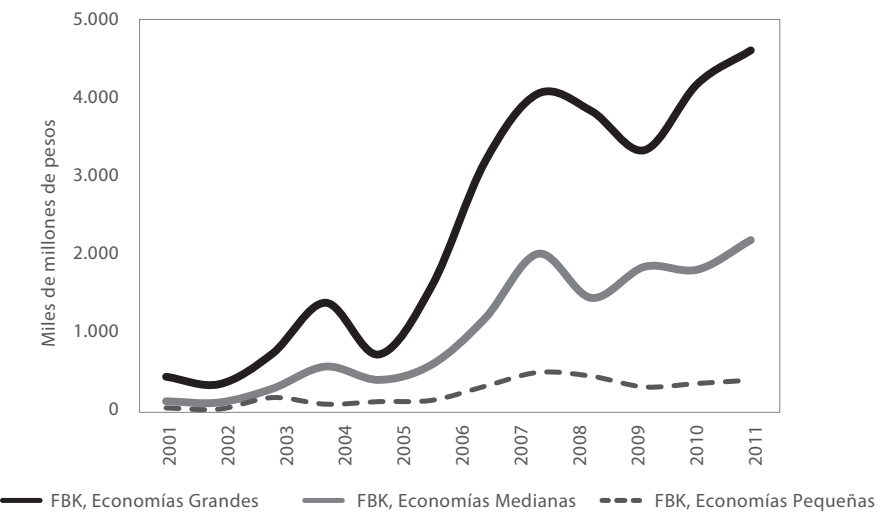

Fuente: elaboración de los autores, con base en información disponible en la página de Internet de Planeación Nacional. 
las economías medianas mostraron un comportamiento mucho más regular durante el periodo a excepción del año 2010, cuya dinámica se frenó.

Por su parte, la formación bruta de capital fijo pública despegó a nivel departamental a partir de 2004, periodo hasta el cual había mantenido unos niveles moderados. Resalta en este comportamiento la aceleración de la inversión pública de las economías grandes, impulsando con apoyo cofinanciado del Gobierno nacional importantes obras de infraestructura, particularmente en obras civiles para el sector transporte.

El año 2002 presentó un resultado atípico para las economías pequeñas en términos de crecimiento de la formación bruta de capital fijo, el cual mostró un crecimiento de $610,3 \%$ respecto a 2001. No obstante, hay que considerar que en valores absolutos, esto significó pasar de una inversión de $\$ 24,1$ miles de millones de pesos en 2001 a \$171,1 miles de millones de pesos en 2002 para el conjunto de los departamentos considerados. Sin tener en cuenta esta irregularidad en la serie, la dinámica de la formación bruta de capital fijo evidencia ciclos cortos volátiles de hasta tres años, con crecimientos pronunciados seguidos de caídas importantes para los tres grupos de entidades territoriales en el periodo analizado, aspecto que evidencia alta homogeneidad regional en su comportamiento.
Comparando los resultados del gasto público y el PIB en evolución en cada uno de los grupos de departamentos, se observa que existe mayor relación en la evolución de dichos agregados en las economías medianas y grandes con algún grado de rezago, debido al impacto que genera el gasto público posteriormente en el PIB. Sin embargo, se evidenció una menor asociación entre el gasto público y el PIB en el grupo de economías departamentales pequeñas, en las cuales se detectó un rezago más marcado, incluyendo periodos en los que no se advierte relación alguna (2005-2007) (gráfica 6). Este comportamiento se constata también en los resultados de la producción departamental para los mismos grupos de entidades territoriales.

En el escenario alternativo, los resultados del modelo muestran la relación entre el crecimiento del $\mathrm{PIB}$, tomado como el logaritmo del producto interno bruto a precios constantes de 2005 (Iconspib, eje de las ordenadas), y el gasto público asumido como el logaritmo de esta variable a precios constantes de 2005 (Iconsgasto, eje de las abscisas). Como se advierte en la gráfica 7, dicha asociación es muy estrecha y altamente correlacionada con respecto al comportamiento que se ha venido presentando en cada uno de los departamentos. El ajuste fitted values es casi perfecto entre estas dos variables y la correlación evidencia el vínculo directo que existe entre los gastos de cada uno de los departamentos en cada año respecto a su dinámica económica.

Gráfica 5.

Formación bruta de capital para todas las economías, serie 2001-2011

(excluye 'outlier' 2002) (Variaciones porcentuales)

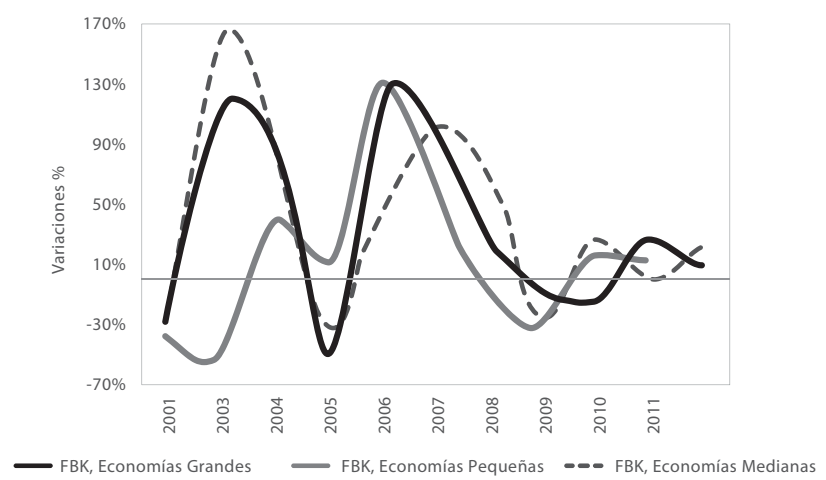

Fuente: elaboración de los autores, con base en las cuentas nacionales del DANE. 
Variación porcentual del gasto público y el PIB. Economías grandes, medianas y pequeñas (2001-2011)

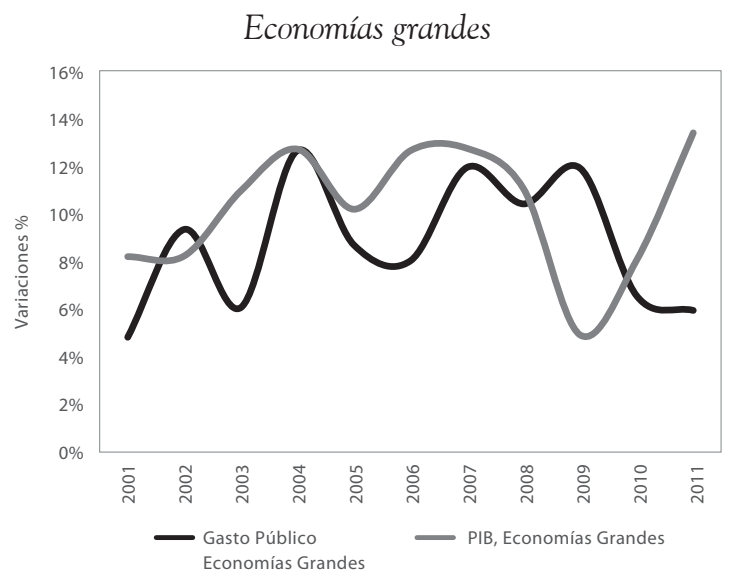

Economías medianas
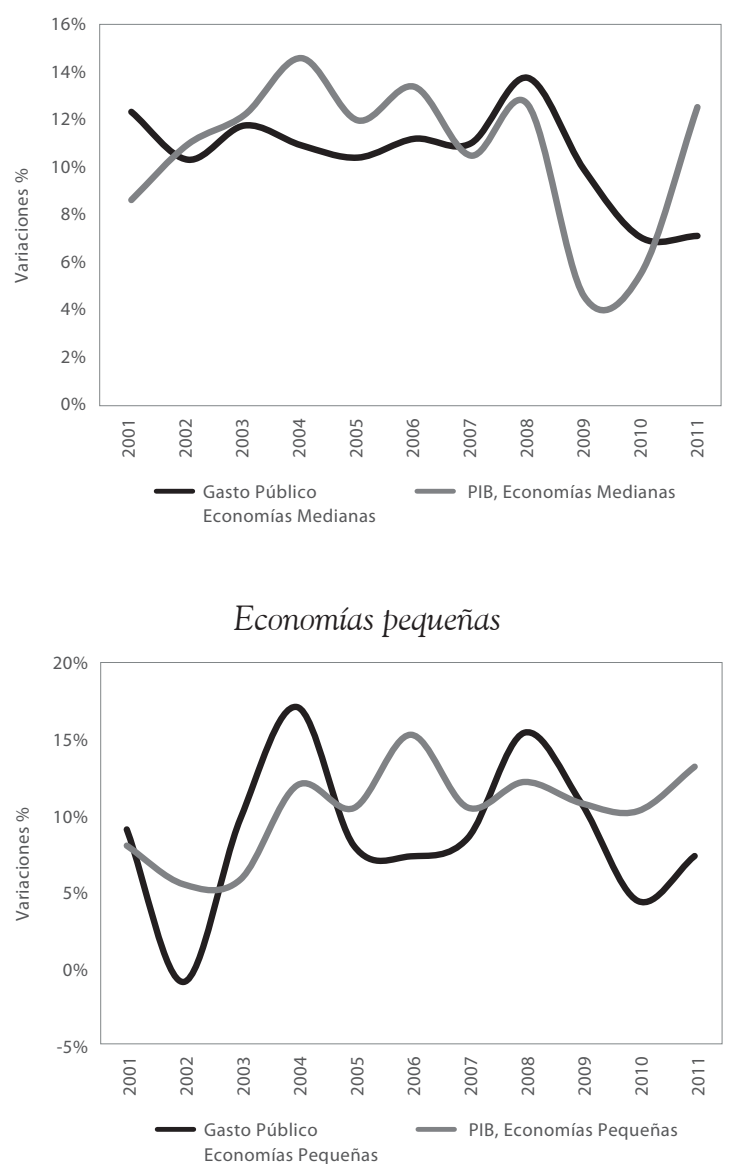

Fuente: elaboración de los autores, con base en las cuentas nacionales departamentales del DANE. 
Respecto a la disyuntiva presentada entre adoptar un modelo tipo panel de efectos fijos o uno basado en efectos aleatorios que represente mejor el impacto que han tenido el gasto y la inversión públicas en el PIB regional, las siguientes tablas muestran los resultados de las dos alternativas, las cuales se someten a valoración selectiva mediante la aplicación de una prueba estadística de consistencia. La tabla 2 muestra los resultados generales del ejercicio de panel data con efectos fijos corregidos por la forma Arellano-Bond comparando con la estimación de cada modelo.

De conformidad con la simulación mediante efectos fijos, se advierte que en los tres grupos de economías regionales el crecimiento económico está directamente relacionado con la inversión y el gasto públicos. El grado de ajuste de este modelo explicado por el $\mathrm{R}^{2}$ o coeficiente de determinación y correlación grado Pearson ( $R^{2}$ efecto within $\left.{ }^{7}\right)$ es de 0,77 ; esto si se establece que el gasto y la inversión pública explican conjuntamente en un $77 \%$ el crecimiento económico de los departamentos en Colombia. ${ }^{8}$

La estimación general obtenida muestra que el gasto público departamental tiene un impacto de 0,69 puntos porcentuales sobre el crecimiento de las economías regionales, en tanto que el efecto estimado de la inversión pública es de tan solo 0,02 puntos porcentuales en el crecimiento. Todos los parámetros son significativos en niveles del $1 \%, 5 \%$ y $10 \%$ de confianza, respectivamente. Con el ejercicio se advierte también que los errores del modelo de la forma de Arellano-Bond no se encuentran correlacionados entre sí, y que visto en las otras estimaciones (efectos fijos y aleatorios) sí lo estaban debido al conjunto de caracterizaciones que podían persistir de manera interna entre estos y/o a variables adicionales por vincular, que no eran objeto de especificación en el modelo.

$7 \quad$ Efectos individuales. Cuando este coeficiente es cercano a uno (1), mayor grado de explicación tienen las variables sobre la variable dependiente del modelo.

8 Otra referencia de bondad de ajuste es la prueba Fisher o prueba global (F), de la cual se constata ser significativa en todos los niveles de análisis del test.

Finanz. polit. econ., ISSN 2248-6046, Vol. 6, No. 1, enero - junio, 2014, pp. 23 - 41 Edición Especial - Economía Regional
Las estimaciones de los coeficientes por efectos aleatorios no son distantes a los obtenidos por la modelación bajo efectos fijos. El grado de ajuste de este modelo explicado por el $\mathrm{R}^{2}$ o coeficiente de determinación y correlación grado Pearson $\left(R^{2}\right.$ efecto within $^{9}$ ) arrojó el mismo resultado e interpretación que en el escenario panel data de efectos fijos $(0,77)$. La estimación con efectos aleatorios expresa que el gasto público departamental tiene un impacto de 1,09 puntos porcentuales sobre el crecimiento de las economías regionales, mayor que en el caso de efectos fijos; mientras que el efecto estimado de la inversión pública es de tan solo de 0,02 puntos porcentuales en el crecimiento, ligeramente menor a la prueba alternativa, pero sí distante a los obtenidos en el modelo instrumentalizado de Arellano-Bond.

Así mismo, los parámetros obtenidos en esta estimación son significativos en todos los casos, en los respectivos niveles de confianza. Esta apreciación se hace efectuando la estimación en un escenario de mínimos cuadrados generalizados (MCG,) bajo el supuesto de la inexistencia de correlación entre los errores del modelo y las variables explicativas, de manera que no existan indicios de endogeneidad. El tratamiento bajo MCG en los modelos de efectos aleatorios busca controlar el problema de heterocedasticidad $^{10}$ de los errores. Bajo efectos fijos, al considerar un término fijo en los departamentos este problema es tratado inmediatamente; por consiguiente, los mínimos cuadrados son consistentes y se corrige el problema de la varianza del error de manera automática.

Con el fin de dirimir la selección del impacto del gasto y la inversión pública sobre el PIB regional mejor especificados, entre las dos alternativas modeladas, se aplica el test de Hausman el cual permite determinar cuáles estimadores (entre fijos o aleatorios) son más consistentes ${ }^{11}$.

9 Efectos individuales. Cuando este coeficiente es cercano a uno (1), mayor grado de explicación tienen las variables sobre la variable dependiente del modelo.

10 La heterocedasticidad implica la variabilidad que puede tomar la varianza del error por efectos del tiempo.

11 En el caso planteado, ninguno de los resultados arrojados deberían ser distintos; intuitivamente, ambos deberían arrojar los mismos parámetros bajo cualquier estimación que se realice. 
Relación entre el crecimiento del PIB departamental

y el crecimiento del gasto público a precios constantes de $2005^{12}$

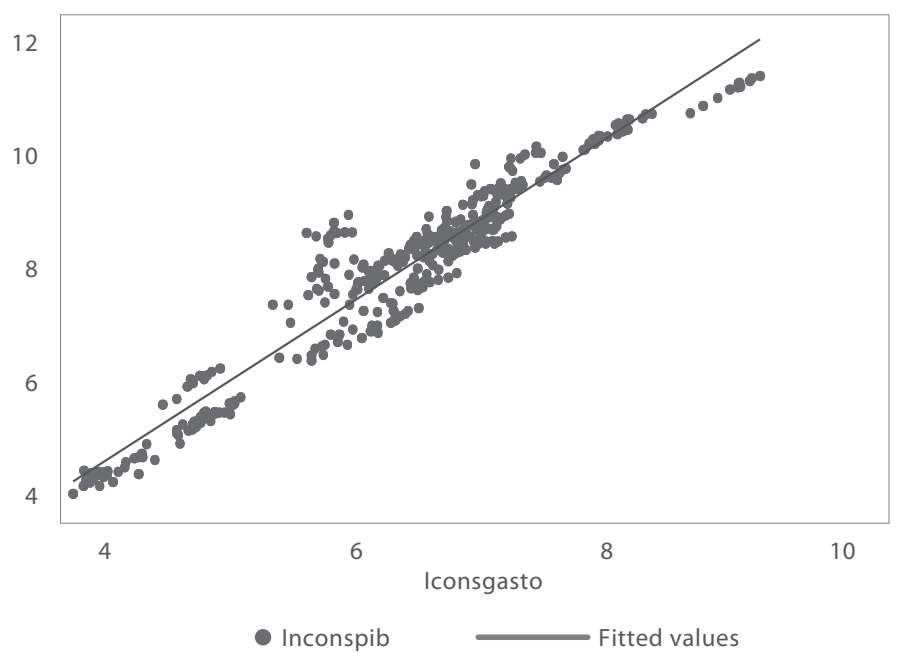

Fuente: elaboración de los autores, con base en las cuentas departamentales elaboradas por el DANE.

Tabla 2.

Regresión de modelo de efectos fijos corregidos, efectos fijos sin corrección y efectos aleatorios

\begin{tabular}{|l|l|l|l|l|}
\hline Coeficientes & $(\mathrm{b})$ & $(\mathrm{B})$ & $(\mathrm{b}-\mathrm{B})$ & sqrt(diag(V_b-V_B) \\
\hline & Efectos Fijos & Efectos Aleatorios & Diferencia & Error estándar \\
\hline Gasto Público & 0,8999746 & 1,098506 & 0,198532 & 0,0191705 \\
\hline Formación bruta & 0,0415872 & 0,0264183 & 0,0151689 & \\
\hline
\end{tabular}

$\mathrm{b}=\quad$ consistente bajo $\mathrm{Ho} \mathrm{y} \mathrm{Ha}$

$\mathrm{B}=\quad$ inconsistente bajo $\mathrm{Ha}$, eficiente bajo $\mathrm{Ho}$

Test: Ho: $\quad$ diferencia en los coeficientes no es sistematica

$$
\begin{aligned}
& \text { chi2 }(2)=(b-B)^{\prime}\left[\left(V_{-} b-V_{-} B\right)^{\wedge}(-1)\right](b-B) \\
& =\quad 107.11 \\
& \text { Prob }>\text { chi2 }=0.0000 \\
& \left(V \_b-V \_B\right. \text { no es positiva semidefinida) }
\end{aligned}
$$

Fuente: elaboración de los autores, con base en las cuentas departamentales del DANE.

12 Los gráficos y cuadros de salida de los modelos fueron elaborados en el software econométrico STATA 12.1 
Test de consistencia de estimadores

\begin{tabular}{|l|l|l|l|l|}
\hline Coeficientes & $(\mathrm{b})$ & $(\mathrm{B})$ & $(\mathrm{b}-\mathrm{B})$ & sqrt(diag(V_b-V_B $)$ ) \\
\hline & Efectos fijos & Efectos aleatorios & Diferencia & Error estándar \\
\hline Gasto Público & 0,8999746 & 1,098506 & 0,198532 & 0,0191705 \\
\hline Formación bruta & 0,0415872 & 0,0264183 & 0,0151689 & \\
\hline
\end{tabular}

$$
\begin{array}{ll}
\mathrm{b}= & \begin{array}{l}
\text { consistente bajo } \mathrm{Ho} \mathrm{y} \mathrm{Ha} \\
\text { inconsistente bajo } \mathrm{Ha} \text {, eficiente bajo Ho }
\end{array} \\
\text { Test: Ho: } & \text { diferencia en los coeficientes no es sistemática }
\end{array}
$$$$
\operatorname{chi} 2(2)=(b-B)^{\prime}\left[\left(V_{-} b-V_{-} B\right)^{\wedge}(-1)\right](b-B)
$$$$
=107.11
$$$$
\text { Prob }>\text { chi2 }=0.0000
$$$$
\text { (V_b-V_B no es positiva semidefinida) }
$$

Ho: $E\left[\mu_{i t}, x_{i t}\right]=0$ Efectos Aleatorios

Ha : $E\left[\mu_{i t}, x_{i t}\right] \neq 0$ Efectos Fijos

Fuente: elaboración de los autores, con base en las cuentas departamentales del DANE.

De conformidad con el estadístico Chi-cuadrado generado en el test de Hausman se concluye que los parámetros del modelo están mejor especificados mediante panel de datos por efectos fijos, dado que con un nivel de significancia del $5 \%$, se rechaza la hipótesis de efectos aleatorios, en la medida que se presentan diferencias sistemáticas ${ }^{13}$ entre los grupos de departamentos objeto de este estudio. Por tanto, la estimación de efectos fijos es más consistente que la arrojada por la de los efectos aleatorios.

\section{DISCUSIÓN}

En cuanto a la metodología y la información disponible, el estudio sobre el impacto que el gasto y la formación bruta de capital fijo públicos tienen actualmente sobre la dinámica de las economías regionales permitió ampliar el espectro de información estadística regional, particularmente de las cuentas nacionales departamentales. Lo anterior gracias a que la estimación de la cuenta de

13 Entre estas diferencias se pueden considerar aquellas que son invariantes entre departamentos, como lo son sus límites geográficos, ubicación espacial, clima, calidad de la tierra, número de habitantes, entre otros factores socioeconómicos relevantes a estos. producción por departamento a nivel de rama de actividad efectuada contribuye al estado del arte en el diseño de un conjunto de indicadores, hasta ahora no disponibles, con los cuales se puede identificar la estructura productiva regional, así como los cambios inter e intra regionales en su composición a lo largo del tiempo.

Si bien dichos resultados pueden orientarse a análisis que se enfoquen en este propósito, este conjunto de datos era necesario para especificar mejor el modelo estadístico que se definió, de manera que sobre un portafolio ampliado de variables que incluyera el valor agregado, la producción y el consumo intermedio regional, y no solo sobre el Producto Interno Bruto, se estimara mejor el efecto que el gasto y la inversión pública en el conjunto de los citados agregados regionales durante el periodo de estudio.

Sin embargo, en la selección y especificación del modelo óptimo, la información estadística disponible juega un papel fundamental y es en este sentido que queda una larga tarea institucional por adelantar. Constituyó una restricción relevante estimar la cuenta de producción regional a partir de una oferta limitada de indicadores y registros administrativos regionales, complementada con las estructuras de sus actividades económicas 
inferidas desde las relaciones técnicas de la función de producción nacional, las cuales no permiten matizar de forma adecuada las asimetrías territoriales. Por tanto, el diagnóstico de necesidades de información y la programación de una oferta ampliada de estadísticas territoriales que surtan la elaboración de las cuentas nacionales departamentales y otros estudios específicos deben ser una directriz que el sistema estadístico nacional debe tomar como prioridad.

Con base en lo anterior y en relación con el modelo estadístico adecuado, su selección en efecto estuvo restringida por la disponibilidad de información, en tanto que un ejercicio de series de tiempo lo era por la extensión de las series. La metodología de panel de datos surgía como alternativa, pero requiere para su aplicación un conjunto específico de variables que capturen los diferentes efectos y comportamientos por observar.

El modelo de panel de datos con efectos fijos definido en virtud al mejor ajuste observado, y adicionando las correcciones vía instrumentos de variables rezagados como lo proponen Arellano y Bond (1991), supone que las economías regionales son heterogéneas, es decir, que existen entre ellas variables y características productivas que las hacen diferentes entre sí, como intuitivamente se consideraría. Por esta razón, el modelo captura particularidades como las que pueden presentarse entre una economía concentrada en la producción de carbón como La Guajira y una región industrial por excelencia como Antioquia, sin perjuicio de la dotación original de factores y recursos que ellas tengan.

La intención de profundizar en las asimetrías de los departamentos de manera individual en virtud del modelo de efectos fijos retornaba las restricciones del proceso a su estado inicial: la disponibilidad de información. La agenda futura de este trabajo llevaría a profundizar en la estimación y/o consecución de datos para variables adicionales de interés por departamento, con el fin de capturar mejor la diversidad regional del crecimiento y el impacto del gasto, y la inversión sobre este.

\section{CONCLUSIONES}

Los resultados obtenidos evidencian un efecto significativo del gasto y la inversión públicos en el crecimiento económico de los departamentos. En tal sentido, se advierte que las economías grandes aprovechan mejor las externalidades positivas del gasto y la formación bruta de capital públicos, y gracias a ello potencian su crecimiento y aumentan así la brecha entre las regiones. El aporte del gasto y la inversión públicos al crecimiento de las economías departamentales pequeñas, si bien es significativo, es menor al que capitalizan las economías grandes y medianas.

Por otro lado, la formación bruta de capital fijo pública muestra un menor impacto en el crecimiento que el propio gasto público. Aquí la eficiencia de la ejecución juega un rol importante, independientemente de la finalidad y su efecto multiplicador. Mientras en Colombia la infraestructura sigue rezagada a pesar del aumento considerable del gasto en inversión identificado desde 2004, la dinámica de este agregado no ha sido constante a largo plazo; por el contrario, ha estado sujeta a ciclos cortos y volátiles comunes entre las economías grandes, medianas y pequeñas que no han garantizado la consolidación de un stock de capital que sea soporte de un crecimiento y desarrollo regional sostenible.

Este documento constituye el primer paso en un proceso tendiente a hacer un aporte en la descripción y análisis de la dinámica económica de los departamentos en Colombia. La agenda futura abarca la estimación de los componentes de la generación del ingreso de los departamentos con el propósito de describir el comportamiento de las demás fuentes de crecimiento locales. Así mismo, adelantar este ejercicio individualizando las estructuras productivas de los departamentos e incorporando información complementaria de tipo local, de manera que se profundice en la identificación de sus heterogeneidades económicas y se analicen los factores que inciden en el desequilibrio económico regional citado en diferentes informes (La República, 2013). 
El diagnóstico obtenido constituye una razón de peso para seguir avanzado en el objetivo propuesto en este trabajo con un mayor grado de profundidad. El más reciente informe sobre competitividad regional sigue mostrando que las economías grandes mantienen la distancia sobre las regiones pequeñas, y que los avances significativos se presentan entre regiones del mismo grupo (Consejo Privado de Competitividad, 2013). Los datos disponibles ponen en evidencia cómo el país padece de sintomatología de "paradoja del crecimiento", según la cual se mantienen divergencias entre un grupo de regiones privilegiadas a las que les favorecen los indicadores de concentración de la riqueza, pero en los que su producto per cápita es bajo y otros en los cuales la pobreza aumenta a lo largo del tiempo y se avanza lentamente en la cobertura de sus índices de necesidades básicas insatisfechas a pesar de ostentar un PIB por persona alto.

\section{REFERENCIAS}

1. Ardila, L. (2004). Gasto público y convergencia regional en Colombia. Revista Ensayos Sobre Política Económica. Banco de la República. Recuperado de http://www.banrep.gov.co/docum/ensayos/pdf/ resu_045-6.pdf

2. Arellano, M. y Bond, S. (1991). Some tests of specification for panel data: Monte Carlo evidence and an application to employment equations. The review of economic studies, 58(2), 277-297.

3. Baltagi, B. H. (2008). Econometric analysis of panel data. Recuperado de http://www.amazon.com/ Econometric-Analysis-Panel-Data-Baltagi/dp/0470518863

4. Consejo Privado de Competitividad (2013). Informe Nacional de Competitividad 2013-2014. Recuperado de http://www.compite.com.co/site/wp-content/uploads/2013/11/CPC INC2013. 2014-Informe.pdf

5. Cortés, A. M. y Pinzón Santos, R. E. (2000). Bases de contabilidad nacional según el SCN 1993. Bogotá: DANE.

6. Departamento Administrativo Nacional de Estadística (DANE) (2010). Dirección de Síntesis y Cuentas Nacionales. Cuentas departamentales, base 2005: resultados y cambios metodológicos. Recuperado de http://www.dane.gov.co/files/investigaciones/pib/departamentales/B_2005/Resultados_cambios_metodologicos.pdf.

7. Departamento Administrativo Nacional de Estadística (DANE) (2013). Cuentas Nacionales Departamentales. Recuperado de http://www.dane.gov.co/index.php/cuentas-economicas/cuentasdepartamentales

8. Domenech, R. (2004) Política fiscal y crecimiento económico. Universidad de Valencia. Recuperado de http://www.uv.es/rdomenec/fiscal_growth.pdf

9. Eurostat (1996). Sistema Europeo de Cuentas 1995. Madrid: INE.

10. Fuentes, N. (2003). Crecimiento económico y desigualdades regionales en México: El impacto de la Infraestructura. Revista Región y Sociedad. Recuperado de http://lanic.utexas.edu/project/etext/ colson/27/fuentes.pdf

11. Gamboa, R. y Messmacher, M. (2002). Desigualdad Regional y Gasto Público en México. Recuperado de http://www.forumfed.org/libdocs/FedMerco02/116-FEME0206-mx-Gamboa-s.pdf

12. Hall, R. E. y Taylor, J. B. (1986). Macroeconomía (3era edición). Barcelona: Antoni Bosh.

13. Infoandina (2013, marzo). Políticas públicas y desarrollo rural en América Latina y el Caribe: el papel del gasto público. Recuperado de http://www.infoandina.org/recursos/pol\%C3\%ADticasp\%C3\%BAblicas-y-desarrollo-rural-en-am\%C3\%A9rica-latina-y-el-caribe-el-papel-del-gasto 
14. Fondo Monetario Internacional (FMI) (2001). Manual de estadísticas de finanzas públicas (MEFP). Recuperado de https://www.imf.org/external/pubs/ft/gfs/manual/esl/pdf/all.pdf

15. Diario La República (2013). Un desequilibrio regional injusto y peligroso. Recuperado de http://www. larepublica.co/un-desequilibrio-regional-injusto-y-peligroso_42718

16. Márquez, M. A., Ramajo, J. y Hewings, G. (2012). Crecimiento regional y capital público: cuantificación del impacto sobre las regiones españolas de shocks negativas en la inversión pública. Recuperado de http://www.aecr.org/web/congresos/2012/Bilbao2012/htdocs/pdf/p526.pdf

17. Mendoza, H. (2012). Impacto del gasto público en la dinámica económica regional. Proyecto de investigación 2013. Universidad Católica de Colombia.

18. Moncayo, E. (2007). Hacia la cobertura universal. Expansión y convergencia de servicios públicos en Colombia 1938-2005. Superintendencia de Servicios Públicos Domiciliarios. Recuperado de http:// www.superservicios.gov.co/c/document library/get file?folderId=65121\&name=DLFE-5874.pdf

19. Mostajo, R. (2000). Gasto social y distribución del ingreso: caracterización e impacto redistribuido en países seleccionados de América Latina y el Caribe. Recuperado de http://www.eclac.org/publicaciones/xml/9/4589/lc11376e.pdf

20. Naciones Unidas et al. (1993). Sistema de cuentas nacionales 1993. Washington D.C.: Naciones Unidas.

21. Naciones Unidas et al. (2008). Sistem of national accounts 2008, Preedited version of Volume 1. Recuperado de http://unstats.un.org/unsd.

22. Parkin, M., Esquivel, G. y Muñoz, M. (2007). Macroeconomía. Versión para Latinoamérica (7a. edición). México: Pearson.

23. Perez, L. R. (1999). Crecimiento y disparidades regionales en Chile: una visión de largo plazo. Estadística y Economía. Recuperado de http://www.ine.cl/canales/chile_estadistico/territorio/publicaciones/ pdf/crecimiento_y_disparidad_regionales.pdf

24. Posada, C. E. y Gómez, W. (2013). Crecimiento económico y gasto público: un modelo para el caso Colombiano. Recuperado de http://quimbaya.banrep.gov.co/docum/ftp/borra218.pdf

25. Rocha, E. y Martínez, H. (2003). Pobreza, crimen y crecimiento regional en Colombia. Archivos de Economía. Departamento Nacional de Planeación. Dirección de Estudios Económicos. Recuperado de http://www.dnp.gov.co/Portals/0/archivos/documentos/DEE/Archivos_Economia/230.pdf

26. Rocha, R. y Vivas, A. (1998). Crecimiento regional en Colombia: ipersiste la desigualdad? Revista de Economía del Rosario. Recuperado de http://www.urosario.edu.co/urosario_files/a1/a152ee6b-be88. 4 fed-95d4-902cd75d2273.pdf

27. Sánchez, O. (2006). Crecimiento Económico Departamental y Migración en Colombia. Archivos de Economía. Departamento Nacional de Planeación. Dirección de Estudios Económicos. Recuperado de http://www.dnp.gov.co/Portals/0/archivos/documentos/DEE/Archivos Economia/317.pdf

28. Shenggen, F. y Neetha, R. (2013, marzo). Public spending in developing countries: trends, determination, and impact. Recuperado de http://people.umass.edu/econ797f/Syllabus\%20and\%20Readings/ Readings/Gender/govspending.strcadj.pdf

29. Social Science Research Network (2012). Public investment and regional growth and convergence: evidence from Greece. Reproducido de http://papers.ssrn.com/sol3/papers.cfm?abstract_id =2153443

30. Vallencilla. (2010). Mediciones del crecimiento económico regional en Colombia. Regiones. Recuperado de http://190.69.156.218:8080/jspui/handle/10988/102

31. Van Tongeren, J. W. (1999). A Systems Approach to National Accounts Compilation. A Technical Report. Studies in Methods. Serie F, 77. Handbook of National Accounting. Department for Economic and Social Affairs Statistics Division. United Nations. New York. 